\title{
Le cœur des affaires de la zone euro.
}

Une analyse structurale et séquentielle des élites économiques transnationales

At the heart of eurozone business: a structural and sequential analysis of the transnational economic elites

Philippe Blanchard, François-Xavier Dudouet et Antoine Vion

\section{(2) OpenEdition \\ Journals}

Édition électronique

URL : http://journals.openedition.org/conflits/19009

DOI : 10.4000/conflits. 19009

ISSN : $1777-5345$

Éditeur :

CECLS - Centre d'études sur les conflits - Liberté et sécurité, L'Harmattan

Édition imprimée

Date de publication : 15 octobre 2015

Pagination : 71-99

ISBN : 978-2-343-07829-8

ISSN : 1157-996X

\section{Référence électronique}

Philippe Blanchard, François-Xavier Dudouet et Antoine Vion, « Le cœur des affaires de la zone euro. »,

Cultures \& Conflits [En ligne], 98 | été 2015, mis en ligne le 15 octobre 2016, consulté le 02 avril 2021

URL : http://journals.openedition.org/conflits/19009; DOI : https://doi.org/10.4000/conflits.19009 


\title{
Le cour des affaires de la zone euro.
}

\author{
Une analyse structurale et séquentielle des élites économiques \\ transnationales
}

\section{Philippe BLANCHARD, François-Xavier DUDOUET, Antoine VION}

Philippe Blanchard est professeur associé de science politique à l'Université de Warwick an Royaume-Uni. Ses travaux et enseignements portent sur la communication politique, les attitudes et politiques écologiques et les méthodes de sciences sociales et politiques, notamment les méthodes statistiques descriptives multivariées, l'entretien, le design de questionnaires, l'analyse de contenu et les méthodes longitudinales. Il co-préside la section Political Methodology de l'European Consortium for Political Research (ECPR). Il enseigne à la Methods School de l'ECPR et préside son conseil scientifique.

François-Xavier Dudonet, sociologue, est chercheur an CNRS et membre de l'IRISSO (Université Paris Dauphine). Ses travaux portent sur la sociologie des dirigeants de grandes entreprises et l'économie politique internationale. Il a développé depuis de nombreuses années une expertise sur les bases de données temporelles et l'analyse prosopographique. Depuis 2012, il anime le réseau thématique "sociologie des élites" au sein de l'Association française de sociologie.

Antoine Vion, Maître de Conférences à Aix-Marseille Université, enseigne la science politique et la sociologie et coordonne un programme de recherche an Laboratoire d'Economie et Sociologie du Travail (UMR CNRS 7317). Ses recherches portent sur les formes de structuration interpersonnelles et inter-organisationnelles du pouvoir en Europe. Il s'est intéresséparticulièrement à la structuration des jumelages et réseaux de villes, à l'organisation de la standardisation industrielle et aux milieux d'affaires de la zone euro. Il est également membre de l'antiAtlas des frontières.

$\mathrm{I}_{\mathrm{n}}^{\mathrm{1}}$ est établi que c'est en Europe, et tout particulièrement en Europe continentale, que les relations d'affaires entre grandes entreprises sont les plus développées ${ }^{1}$. Il reste néanmoins à qualifier précisément ce phénomène.

1. Voir Fennema M., International networks of banks and industry, The Hague, Martinus 
Longtemps, la question des milieux d'affaires transnationaux a été l'apanage d'une pensée marxiste, qui envisageait l'existence de la classe capitaliste transnationale de manière théorique, soit à partir d'un enracinement étatique ${ }^{2}$, soit contre les États ${ }^{3}$. L'une des principales critiques faite à cette tradition fut de manquer d'épaisseur empirique ${ }^{4}$, à propos des trajectoires et pratiques effectives des dirigeants de grandes entreprises ${ }^{5}$ ou des modes de socialisation transnationale : il ne suffit pas de parler de classe capitaliste transnationale pour la faire exister, encore faut-il la mesurer concrètement ${ }^{6}$. C'est dans ce contexte que l'analyse de réseaux appliquée aux conseils d'administration, aussi appelée interlocking directorate studies ${ }^{7}$, a été mobilisée pour essayer de mesurer plus précisément en quoi les dirigeants de grandes entreprises sont en relation les uns avec les autres au plan transnational.

Les premières applications notables furent tentées dès les années 1980 8, mais ce type d'études prit son essor à partir des années 2000. Il fait alors clairement ressortir l'existence de relations transnationales entre les plus grandes firmes mondiales, mais conteste l'idée d'une classe capitaliste transnationale en soi et pour soi ${ }^{9}$. La comparaison des réseaux nationaux et transnationaux

Nijhoff Publishers, 1982 et Carroll W., The Making of a Transnational Capitalist Class. Corporate Power in the 21st Century, Zed Books, 2010.

2. Cox R., Production, Power and World Order, New York, Columbia University, 1987.

3. Robinson W. I., Harris J., "Towards a global ruling class? Globalization and the transnational capitalist class", Science E Society, 64, 2000. pp. 11-54.

4. C'est au fond le cœur de la critique de Mills et plus généralement de la tradition américaine des élites à l'égard du marxisme et des approches en termes de classes sociales. Sur ce point voir la note de Jean-Claude Passeron dans sa présentation de Schumpeter J., Impérialisme et classes sociales, Paris, Flammarion, 1984, p. 21, note 15.

5. Wagner A.-C., Les classes sociales dans la mondialisation, Paris, La Découverte, 2007.

6. Voir Carroll W., Fennema M., "Is There a Transnational Business Community?", International Sociology, 17, 2002, pp. 393-419; Carroll W., Fennema M., "Problems in the Study of the Transnational Business Community: A Reply to Kentor and Jang", International Sociology, 19, 2004, pp. 369-378 ; Carroll W., Carson C., "The network of global corporations and elite policy groups. A structure for transnational capitalist class formation”, Global Networks, 3/1, 2003, pp. 29-57. Les penseurs marxistes ne sont pas restés insensibles à cette critique, voir Murray G., "Australia's ruling class. A local elite a transnational capitalist class or bits of both?”, in Murray G., Scott, J. (eds.), Financial elites and transnational business. Who rules the world?, Cheltenham, Edward Elgar, 2012, pp. 217-249 et Burris V., Staples C.L., "In search of a transnational capitalist class. Alternative methods for comparing director interlocks within and between nations and regions", International Journal of Comparative Sociology, 53/4, 2012, pp. 323-342.

7. Inventée au début du XXe siècle pour évaluer le pouvoir des banques sur l'industrie et dénoncer les ententes industrielles, la démarche vise à mesurer l'imbrication des conseils d'administration les uns avec les autres par l'intermédiaire des administrateurs qu'ils ont en commun, d'où l'expression Interlocking directorate proposée par le Comité Pujo dès 1913. Longtemps l'apanage d'enquêtes publiques ou de quelques intellectuels radicaux, cette démarche n'a vraiment pris son essor qu'à partir des années 1960-1970, moment où la diffusion de l'informatique dans les universités favorisa grandement ce type d'études. L'engouement concerna cependant surtout les États-Unis et l'Europe du Nord. La France et l'Europe du Sud sont restées jusqu'à récemment à l'écart du mouvement.

8. Fennema M., op. cit. Voir également Stockman F. N., Ziegler R., Scott J. (eds.), Networks of Corporate Power. A Comparative Analysis of Ten Countries, Cambridge, Polity Press, 1985.

9. Voir Carroll W., Fennema M., op. cit. ; Carroll W., Carson C., op. cit. Voir aussi Nollert M., "Transnational Corporate Ties. A Synopsis of Theories and Empirical Findings", Journal of 
permet tout au plus de parler d'une communauté d'affaires transnationale (transnational business community) en voie d'émergence ${ }^{10}$. Bien que ces travaux ne soient pas exempts de critiques, notamment sur le plan méthodologique ${ }^{11}$, ils ont le mérite de fournir une mesure empirique d'un phénomène qui en manquait singulièrement. Les interlocking directorate studies ne sont certes pas la seule manière de mesurer l'internationalisation des dirigeants économiques. On peut aussi faire la prosopographie des dirigeants à travers leur nationalité, leurs études, ou encore leur trajectoire professionnelle ${ }^{12}$. Mais il ne faudrait pas, comme le fait Hartmann ${ }^{13}$, opposer les deux approches. Si la prosopographie nous renseigne utilement sur la composition des groupes, comme la proportion d'étrangers à la tête des firmes, elle dit peu sur l'architecture des relations transnationales entre les firmes et leurs dirigeants. Constater qu'une direction est fortement internationalisée, comme celle du suisse $\mathrm{ABB}$, qui comprend pas moins de dix nationalités parmi les 20 membres de sa direction ${ }^{14}$, ne renseigne pas sur les relations que ces dirigeants entretiennent avec leurs homologues des autres firmes. Or c'est aussi sur ce plan que se joue l'internationalisation des milieux d'affaires.

En fait, la critique majeure que l'on peut faire à l'analyse de réseaux n'est pas tant de « rater» la mesure du phénomène transnational que de se concentrer presque exclusivement sur les relations inter-firmes au détriment des relations interindividuelles, c'est-à-dire du réseau formé par les dirigeants euxmêmes. Cela conduit à une appréciation très organisationnelle du phénomène. On passe ainsi à côté d'une connaissance sociologique des hommes et des femmes d'affaires transnationaux, ce qu'ils sont, d'où ils viennent, et comment ils se socialisent. Pour Yves Dezalay et Michael Rask Madsen ${ }^{15}$, les agents dans ce type d'études ne seraient « jamais situés - ou de manière très accessoire - dans les espaces nationaux où ils ont acquis des ressources, familiales, scolaires, professionnelles ou politiques, susceptibles d'influencer leurs parcours ultérieurs dans des espaces transnationaux, notamment en ce qui concerne

World-systems Research, XI/2, 2005, pp. 289-314 ou Dudouet F.-X., Grémont E., Vion A., "Transnational business network in the Eurozone: a focus on four major stock exchange indices”, Murray G., Scott J. (eds.), op. cit., pp. 124-145.

10. Carroll W., Fennema M., op. cit.

11. Vion A., Dudouet F.-X., Grémont E., “The Eurozone's Corporate Elite at the Cliff Edge (2005-2008): A New Approach to Transnational Integration”, In Morgan G., Quack S., Hirsch P. (eds.), Elites on trial, Research in the Sociology of Organizations Series, London, Emerald, 2015.

12. Dudouet F.-X., Joly H., «Les dirigeants français du CAC 40 », Sociologies Pratiques, 21, 2010, pp. 35-48 ; Dudouet, F.-X., Grémont E., Joly H., Vion A., « Retour sur le champ du pouvoir économique en France. L'espace social des dirigeants du CAC 40 », Revue Française de Socio-Economie, 13, 2014, pp. 23-48.

13. Hartmann M., "Internationalisation et spécificités nationales des élites économiques », Actes de la recherche en sciences sociales, 190/5, 2011, pp. 10-23.

14. Conseil d'administration et comité exécutif réunis : 3 Américains, 2 Allemands, 1 Brésilien, 1 Chinois, 3 Finnois, 2 Français, 1 Irlandais, 1 Italien, 2 Suédois, 2 Suisses, 1 Suisso-Allemand, 1 Suisso-Suédois, ABB Corporate Governance, http://new.abb.com/about/corporate-governance (Page consultée le 22 avril 2015).

15. Op. cit. 
leurs pratiques ou leurs choix stratégiques 16 ». Cette critique fait écho à tout un courant de la sociologie française qui, depuis le milieu des années 1990, tente de saisir les phénomènes transnationaux et européens à partir des outils de la sociologie classique ${ }^{17}$. Ces travaux insistent sur les propriétés sociales, la mobilité et le travail d'agents sociaux particuliers dans la construction des espaces transnationaux, qu'il s'agisse des professionnels du droit ${ }^{18}$, de la sécurité ${ }^{19}$, de la comptabilité ${ }^{20}$, de l'Europe politique ${ }^{21}$, des organisations patronales 22 et syndicales 23 ou encore des dirigeants de grandes entreprises ${ }^{24}$. Nombre de ces recherches réfléchissent en termes de champs, notamment celui du pouvoir, dans un contexte, si ce n'est transnational, du moins européen ${ }^{25}$. Une autre dimension de cette sociologie est d'affronter directement la

16. Dezalay Y., Rask Madsen M., «Espaces de pouvoirs nationaux, espaces de pouvoir internationaux ", in Cohen A., Lacroix B., Riutort P. (eds.), Nouveau manuel de science politique, Paris, La Découverte, 2011, chapitre XII, 2.

17. Devin G., "Norbert Elias et l'analyse des relations internationales ", Revue française de science politique, 45/2, 1995, pp. 205-327 ; Bigo D., Polices en réseaux. L'expérience européenne, Paris, Presses de Sciences Po, 1996 ; Dezalay Y., Garth B., Dealing in virtue. International commercial arbitration and the emergence of a new international legal order, Chicago, University of Chicago Press, 1996 ; Wagner A.-C., Les nouvelles élites de la mondialisation. Une immigration dorée en France, Paris, PUF, 1998.

18. Dezalay Y., Marchands de droit: La restructuration de l'ordre juridique international par les multinationales du droit, Paris, Fayard, 1992 ; Cohen A., "Constitutionalism without constitution. Transnational elites between political mobilization and legal expertise in the making of a constitution for Europe (1940s- 1960s)", Law \& Social Inquiry, 32/1, 2007, pp. 109-135; Vauchez A., "The force of a weak field: law and lawyers in the government of the European Union (for a renewed research agenda)", International Political Sociology, 2/2, 2008, pp. 128144 ; Cohen A., Vauchez A., "The social construction of law: The European Court of Justice and its legal revolution revisited", Annual Review of Law and Social Science, 7, 2011, pp. 417431.

19. Bigo D., op. cit.

20. Dezalay Y., "Accountants as 'new guard dogs' of capitalism: Stereotype or research agenda?", Accounting, Organizations and Society, 22/8, 1997, pp. 825-829; Ramirez C., "How Big Four audit firms control standard-setting in accounting and auditing", in Huault I., Richard C. (eds.), Finance: The Discreet Regulator: How Financial Activities Shape and Transform the World, New York, Palgrave Macmillan, 2012, pp. 40-58.

21. Georgakakis D., Rowell J. (eds.), "The Field of Eurocracy, Mapping EU Actors and Professionals", New York, Palgrave Mcmillan, 2013 ; Michon S., Les équipes parlementaires des eurodéputés. Entreprises politiques et rites d'institution, Larcier-Promoculture, 2014.

22. Pageaut A., "The current members of the European Round Table: A transnational club of economic elites", French Politics, 2010, 8, pp. 275-293 ; Michel H. (ed.), Représenter le patronat européen. Formes d'organisation patronale et modes d'action européenne, Bruxelles, P.I.E. Peter Lang, 2013 ; Laurens S., " Make it EU friendly : Les entrepreneurs du "patronat européen” face aux effets de la concurrence économique », Sociétés Contemporaines, 1/89, 2013, pp. 17-46.

23. Hassenteufel P., Pernot J.-M., "Les syndicats à l'épreuve de l'Europe ", Politique européenne, numéro spécial, 27, 2009; Wagner A.-C., «Les syndicalistes de la Confédération européenne des Syndicats : un capital européen spécifique ? " in Georgakakis D. (ed.), Le champ de l'Eurocratie. Une sociologie politique du personnel de l'Union européenne, Paris, Economica, 2012, pp. 241-256.

24. Dudouet F.-X., Grémont E., Pageault A., Vion A., "European Business Leaders: A Focus on the Upper Layers of the European Field Power", in Georgakakis D., Rowell J. (eds), op. cit., pp. 202-225.

25. Georgakakis D., Rowell J. (eds.), op. cit. ; Bigo D., "Pierre Bourdieu and International Relations: Power of Practices, Practices of Power", International Political Sociology, 5/3, 2011, pp. 225-258 ; Cohen, A., "Bourdieu hits Brussels: the genesis and structure of the European field of power", International Political Sociology, 5/3, 2011, pp. 335-339 ; 
tension entre national et transnational, notamment en associant étroitement l'ancrage national des agents à leur mobilité transnationale. Les concepts « d'agent double 26 », de « passeurs 27 » ou encore d'acteur-clef 28 viennent ainsi rendre compte des imbrications du national et du transnational et du rôle déterminant qu'y jouent certains individus. Pour Dezalay et Garth ${ }^{29}$, les agents doubles sont tout à la fois inscrits dans la reproduction des hiérarchies sociales de leur espace de pouvoir national et parties prenantes de la constitution d'un champ du pouvoir plus large, dans la mesure où ils opèrent la transcription des dogmes économiques et des règles juridiques de pays tiers. En effet, les relations transnationales ne reposent pas sur des échanges symétriques, mais peuvent recouvrir des logiques de domination comme l'absorption d'un milieu d'affaires national par un autre ${ }^{30}$. Dès lors, l'identification et la description fine des acteurs qui structurent cet espace transnational contribuent à mettre au jour le phénomène transnational lui-même. C'est la raison pour laquelle nous proposons de nous attacher aux dirigeants de grandes entreprises qui structurent le cour du milieu d'affaires de la zone euro.

L'étude que nous exposons ici procède en opérations de recherche successives qui, si elles sont fondées sur un même matériau, recourent à des méthodes différentes : analyse de réseau, statistique descriptive, analyse séquentielle. C'est pourquoi il nous paraît important de présenter notre démarche (1) avant d'analyser la structure du réseau d'affaires transnational et d'y identifier les interlockers transnationaux (2). Nous regarderons ensuite un certain nombre de leurs propriétés sociales (3), puis leurs parcours professionnels (4), pour enfin interroger leur carrière en fonction du type d'activité exercée (5).

\section{Comment étudier les élites économiques de la zone euro?}

L'espace transnational que nous avons choisi d'examiner est celui formé par les directions imbriquées des grandes entreprises de la zone euro en 2008 (voir ci-dessous pour la définition du corpus). Nous identifions les individus affiliés à deux institutions étrangères l'une à l'autre et examinons, d'une part le réseau qu'ils forment entre eux, d'autre part un certain nombre d'informations prosopographiques comprenant le sexe, l'âge, la nationalité, la formation et la trajectoire professionnelle. L'analyse de réseau intervient en première instance pour saisir le degré de proximité des acteurs transnationaux : forment-ils un

Dezalay Y., "Les usages internationaux du concept de champ juridique », Actes de la recherche en sciences sociales, 5/200, 2013, pp. 56-69.

26. Dezalay Y., Garth B., op. cit.

27. Devin G., "La diplomatie d'État vue par les ONG », Autrement. Série mutations, 213, 2002, pp. 100-109.

28. Dudouet F-X., Le grand deal de l'opium : histoire du marché légal des drogues, Paris, Syllepse, 2009.

29. Op. cit.

30. Vion A. et al., op. cit. 
espace dense d'interconnaissances ou sont-ils isolés les uns des autres, œuvrant seulement à mettre en relation des entreprises deux à deux ? De la réponse dépend la consistance de l'espace transnational étudié. L'analyse prosopographique, de son côté, mesure les points de rapprochement entre types de carrière et les points d'éloignements liés aux tropismes nationaux des formations, donc de la reproduction des élites.

Enfin, à l'heure de la "financiarisation ", nous reprendrons à notre compte la question fondatrice des interlocking directorate studies sur le rôle joué par la finance dans la structuration des milieux d'affaires. Plus d'un siècle après ses premières formulations, elle n'a toujours pas reçu de réponse définitive, si ce n'est la tendance des firmes financières, banques et assurances en particulier, à occuper une position plus centrale que les autres, et donc à jouer un rôle plus structurant, dans les réseaux interlocks ${ }^{31}$. Toutefois, assez peu de travaux se sont penchés sur le profil des interlockers eux-mêmes, et encore moins au niveau transnational. Pourtant il y a matière à s'interroger sur le rôle que jouent les financiers dans la structuration des milieux d'affaires transnationaux, en particulier au sein d'une même zone monétaire. Si on admet que la fonction première de la finance est d'émettre et de faire circuler la monnaie ${ }^{32}$, alors il n'y a plus guère de doute sur l'intérêt d'une telle interrogation. Dans une enquête qui se voulait exploratoire, Dudouet et al. 33 avançaient que les financiers étaient proportionnellement mieux représentés que les autres types de dirigeants au sein des interlockers transnationaux, mais il convient d'approfondir ce constat, notamment en augmentant le nombre de cas étudiés et en construisant le profil des acteurs dans la profondeur historique de leurs trajectoires.

L'analyse de réseau est donc mobilisée dans un premier temps pour construire l'espace transnational du milieu des affaires de la zone euro (à partir duquel on peut identifier ses chevilles ouvrières) que nous appelons interlockers transnationaux, ou acteurs-clef. Ces acteurs-clef sont ensuite étudiés de manière réticulaire et prosopographique afin d'en mieux saisir la cohésion, les similitudes et les différences. Enfin, nous interrogeons le poids des dirigeants issus de la finance au sein de ce petit groupe. Nous construisons les différents profils à partir de la trajectoire professionnelle de chaque acteur, examinée à l'aide de l'analyse séquentielle. Les trajectoires professionnelles sont

31. Bearden J., Atwood W., Freitag P., Hendricks C., Mintz B., Schwartz M., "The Nature and Extent of Bank Centrality in Corporate Networks", Paper to the American Sociological Association, 1975 (document réprographié) ; Scott J., Corporate Business and capitalist classes, Oxford, Oxford University Press, 1997 ; Dudouet F.-X., Grémont E., Vion A., « La “centralité des banques” dans les réseaux d'administrateurs. Les conditions sociale de l'émission monétaire ", Revue Française de Socio-Economie, Hors Série, 2015, pp. 37-50.

32. Schumpeter J. A., Théorie de la monnaie et de la banque. Tome I : L'essence de la monnaie, Paris, L'Harmattan, 2005.

33. Dudouet F.-X., Grémont E., Vion A., «La centralité des financiers dans les mondes d'affaires européens ", in Mendez A., Tchobanian R., Vion A. (eds.), Travail et compétences dans la mondialisation, Paris, Armand Colin, 2011, chapitre 2. 
établies depuis la fin des études jusqu'à 2008, date de clôture de l'étude. Deux critères sont particulièrement mobilisés dans l'analyse séquentielle : le ou les secteur(s) dans lesquels l'individu aura fait sa carrière et les fonctions financières exercées en dehors du secteur de la finance (par exemple, directeur financier d'une firme industrielle ou haut fonctionnaire chargé des questions financières). Nous construisons finalement une typologie visant à identifier des profils contrastés de trajectoire au sein de l'élite économique européenne.

Les liens transnationaux s'élaborent à partir de contextes nationaux qu'il convient d'identifier, ne saurait-ce que pour mesurer la réalité du phénomène transnational. À cette fin, nous nous basons sur les grands indices boursiers nationaux en considérant que les dirigeants des entreprises qui composent ces indices forment le sommet de l'élite économique d'un pays donné. Nous faisons ce choix pour de plusieurs raisons. En premier lieu, lorsqu'on mène une analyse de réseaux sur un réseau complet, la délimitation du corpus est cruciale. Cette question n’a guère été posée pour elle-même dans les études interlocks, la plupart d'entre elles se contentant de reprendre la liste des plus grandes firmes telle que fournie par des magazines à grand tirage comme Fortune. Pourtant, il est hardi de postuler, même sous forme d'hypothèse, qu'une liste de firmes regroupées par la seule vertu de la taille de leur chiffre d'affaires soit l'indicateur de l'existence d'un milieu d'affaires. En fait, la très grande majorité des études interlocks s’inscrivent dans la lignée de C. Wright Mills ${ }^{34}$ en assumant la cohésion a priori de l'élite des affaires (corporate elite), mais elles ne s'interrogent pas sur la constitution du corpus ${ }^{35}$. La cohésion des dirigeants de grandes entreprises n'est pas discutée en amont de l'analyse de réseaux elle-même. À l'inverse, nous choisissons les indices boursiers pour leur valeur sociologique. Ils sont, certes, des artefacts, mais, à la différence des classements par la presse, ils sont des produits indigènes typiques de l'activité capitaliste moderne, la manière dont les grandes entreprises, non seulement organisent leur présence sur les marchés financiers, mais aussi se donnent à voir et se mesurent entre elles. Rao et al. ${ }^{36}$ ont montré qu'ils configurent si directement les identités et les hiérarchies des entreprises que celles-ci apportent la plus grande attention à leur stratégie d'affiliation. Loin de se résumer à des agrégats économiques, les indices boursiers sont ainsi des réalités sociales sui generis dont il s'agit de dévoiler la structure.

Deuxièmement, l'entrée par les indices boursiers résout la délicate question de l'identification de la «nationalité » des entreprises multinationales 37. Trop d'études s'en tiennent à la localisation du siège social, sans vérifier que ce siège puisse être une simple boîte aux lettres, comme EADS aux Pays-Bas.

34. Mills C. W., The Power Elite, Oxford University Press, 1999 (1956).

35. Vion A. et al., op. cit.

36. Rao H., Davis G., Ward A., "Embeddedness, social identity and mobility. Why firms leave the NASDAQ and join the New York stock exchange”, Administrative Science Quarterly, 45/2, juin 2000, pp. 268-292. 
Par ailleurs, en se concentrant sur la taille des entreprises, elles surreprésentent certains pays, comme les États-Unis, et passent par pertes et profits les milieux d'affaires constitués par des entreprises de taille beaucoup plus réduite. Nous retrouvons ici la dimension politique de l'encastrement chère à Polanyi 38 qui, à la suite de Weber ${ }^{39}$, s'intéressait aux processus de monopolisation du pouvoir financier à partir de capitaux spécifiques, comme la connaissance des techniques financières et le prestige social au sein des places boursières. De fait, le maintien de " places financières » et d'indices boursiers nationaux à l'heure de la dématérialisation des marchés financiers et du déracinement de plus en plus prononcé des grandes entreprises peut apparaître comme un archaïsme, mais aussi résonner comme la manifestation objective d'un milieu social qui ne s'est pas encore totalement fondu dans la mondialisation ${ }^{40}$. Il fait bien longtemps que les indices boursiers ne révèlent plus rien de la santé économique des pays où ils sont implantés. Leur résistance, envers et contre tout, y compris leur incapacité à jouer leur rôle de baromètre économique sur le plan national, dévoile le caractère éminemment social de ces institutions financières. Il atteste de la permanence des milieux d'affaires nationaux et de leur ancrage politique.

Troisièmement, le fait de regrouper les indices boursiers d'une même zone monétaire rejoint l'idée que le financement des entreprises repose fondamentalement sur divers mécanismes d'émission et de circulation monétaires. Ce financement se fait bien au-delà et en deçà des formes immédiates et évidentes de relations que sont la dette et la participation au capital, c'est-à-dire à travers des aspects beaucoup plus essentiels pour le financement des entreprises que sont les opérations de tenue de compte, d'affacturage et surtout d'intermédiation boursière ${ }^{41}$. Nous avons suggéré que les débats sur la centralité bancaire dans les réseaux interlocks ont bien trop laissé en point aveugle les mécanismes de l'émission et de la circulation monétaire ${ }^{42}$.

\section{Le réseau d'administrateurs de la zone euro}

L'une des manières les plus éprouvées d'évaluer la cohésion d'un milieu d'affaires est de mesurer le degré d'imbrication des directions des entreprises le composant. Cela consiste principalement à diviser le nombre de directions réellement imbriquées par le nombre total d'imbrications possibles. On éta-

37. Vion A. et al., op. cit.

38. Polanyi K., La Grande transformation. Aux origines politiques et économiques de notre temps, Paris, Gallimard, 1983 (1944).

39. Weber M., La Bourse, Paris, Allia, 2010 (1894 et 1896).

40. Ainsi en 2009, alors que la quasi-totalité des entreprises du CAC 40 réalisait la majorité de leur chiffre d'affaires hors de France, seules trois d'entre elles avaient des organes de direction majoritairement composés d'étrangers.

41. Hilferding R., Le capital financier. Étude sur le développement récent du capitalisme, Paris, Éditions de Minuit, 1970, [1910].

42. Dudouet F.-X., Grémont E., Vion A., «La “centralité des banques”... », op. cit. 
blit pour se faire une matrice associant à chaque entreprise plusieurs dirigeants. À partir de cette matrice on trace un premier réseau dit two-modes où sont représentés dirigeants et entreprises. De ce premier réseau on peut en déduire deux autres : un premier réseau de relations entre les entreprises dont les liens sont les dirigeants, un second réseau de relations interpersonnelles dont les liens sont les entreprises. Bien que premier, le réseau two-modes est rarement utilisé, notamment parce que les mesures sont plus difficiles à effectuer et à interpréter. C'est le réseau inter-firmes qui est le plus souvent mobilisé par les études interlocks pour rendre compte de la cohésion des milieux d'affaires. Notre corpus se compose des membres de conseils d'administrations, de surveillance ${ }^{43}$ et de directoires ainsi que des directeurs généraux et directeurs généraux délégués (ou leurs équivalents) des entreprises cotées dans les cinq principaux indices de la zone euro - l'AEX 25 (Pays-Bas), le BEL 20 (Belgique), le CAC 40 (France), le DAX 30 (Allemagne), et le MIB 40 (Italie) ${ }^{44}$. La base de données, constituée par nos soins, couvre les entreprises et leurs dirigeants année après année de 2005 à 2008. Pour la présente étude, nous avons retenu la dernière année couverte. Pour éviter les doublons dans le cas d'enregistrement dans plusieurs indices, nous avons affilié les compagnies en privilégiant la nationalité du directeur général. Nous avons ainsi affilié Dexia au BEL 20, Unibail-Rodamco à l'AEX et ST Microelectronics au MIB 40. Nous avons aussi soustrait Arcelor-Mittal du fait de sa multi-affiliation et de la nationalité indienne de son directeur général. Pour 2008, notre corpus rassemble 148 entreprises et 2011 dirigeants.

Avant d'examiner plus précisément les acteurs qui font l'Europe des affaires, il convient de décrire le réseau sur lequel nous nous basons pour les identifier. Le graphe 1 offre une représentation du réseau inter-firmes. Il regroupe 148 sociétés réparties dans cinq indices nationaux et les liens qu'elles entretiennent entre elles à la fin de l'année 2008 ( $c f$. encadré). Premier constat, la grande majorité des entreprises ( $92 \%$ ) fait partie d'une seule et même composante, deux contribuent à former un réseau secondaire et sept sont des isolats complets. Le diamètre du réseau 45 pour la composante principale est de sept, ce qui peut être interprété comme une assez bonne connectivité. Toutefois, il y a loin à déduire de ces premières mesures un milieu des affaires européen intégré. La densité du réseau, principal indicateur de cohésion, est assez faible $(5 \%)$ et systématiquement inférieure à celui des réseaux nationaux : $9 \%$ pour le BEL, $11 \%$ pour le MIB, $12 \%$ pour l'AEX, $21 \%$ pour le CAC et $25 \%$ pour le DAX. Il est délicat de comparer des scores de densité entre des réseaux de taille différente, néanmoins ces mesures laissent penser

43. Nos données sur les conseils d'administration incluent les censeurs, représentants de l'État et/ou des employés et les observateurs, mais pas les présidents et secrétaires honoraires.

44. Nous avons choisi à dessein les pays fondateurs des Communautés économiques européennes et un ensemble géographique qui représente environ $75 \%$ du PIB de la zone euro. Nous sommes conscients du fait que les indices espagnol et autrichien seraient un complément utile à notre étude, quoique non indispensable.

45. Distance maximale entre deux nœuds. 
que le réseau européen est plus structuré par la « mise en réseau » des réseaux nationaux que par une parfaite intégration transnationale où les relations entre firmes seraient indépendantes de leurs ancrages nationaux ${ }^{46}$. Ceci est confirmé par le fait que près des trois quarts des liens (760) sont nationaux, contre un quart internationaux (254). De même, si la majorité des firmes entretiennent au moins un lien transnational (85 sur 148), seules 14 présentent un solde transnational-national positif, dont trois seulement n'ayant que des liens transnationaux. Encore cette ouverture à l'international n'est-elle pas équivalente pour tous les indices : $81 \%$ des sociétés du CAC possèdent au moins un lien transnational, contre $28 \%$ pour celles du MIB 47.

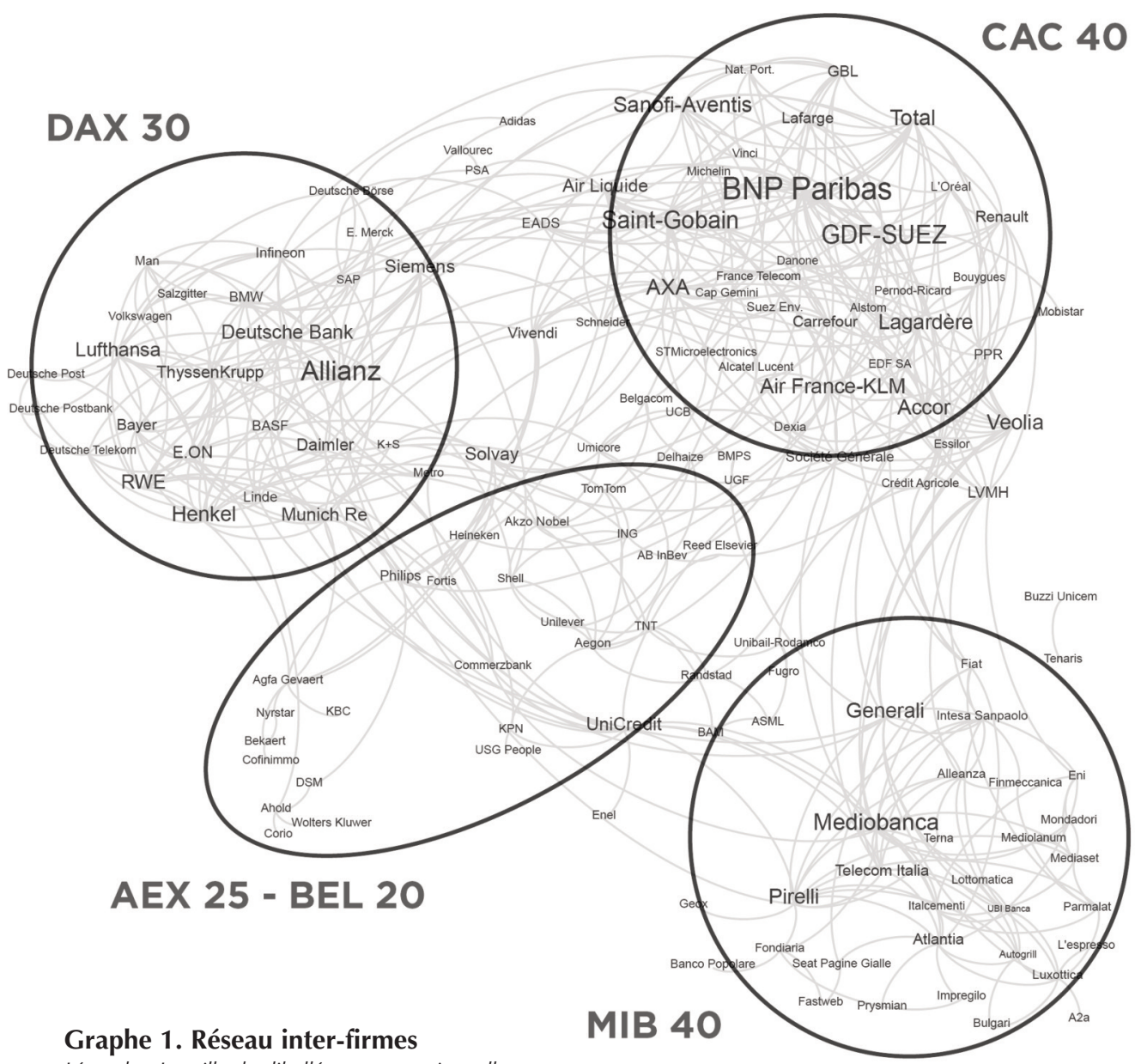

Légende: La taille des libellés est proportionnelle

à la centralité de degré de la firme

(nombre de sociétés en relation directe avec la firme).

46. Le test du Chi2 appliqué au même corpus pour les années 2006 et 2007 confirme la relative indépendance des réseaux nationaux Voir Dudouet F.-X., Grémont E., Vion A., "Transnational business networks...", op. cit.

47. Vion A. et al., op. cit. 
Le milieu des affaires de la zone euro n'est donc pas un espace intégré et homogène mais plutôt un patchwork de milieux nationaux diversement structurés et faiblement liés les uns aux autres. Sous cet aspect, la question des dirigeants qui font le lien entre les différents indices devient cruciale. Contribuant à ouvrir des espaces sociaux les uns sur les autres, ces acteurs sont les chevilles ouvrières du phénomène observé. Sur les 2011 dirigeants recensés, 284 contribuent à mettre en relation les 148 sociétés étudiées et 70 seulement de manière transnationale. Le réseau européen repose donc sur un très petit nombre d'individus qui en sont les véritables acteurs-clefs.

Ces 70 agents représentent pour nous le cœur du milieu d'affaires transnational de la zone euro au sens strict, ce que les anglo-saxons nommeraient, à la suite de Michael Useem, le transnational inner circle ${ }^{48}$. Reprenant l'expression à Mills ${ }^{49}$, Useem avait proposé l'idée que l'identification d'un cercle d'acteurs dominant l'univers économique - The Inner Circle - pouvait être la manifestation objective d'un groupe social plus vaste dont il assurerait justement la cohésion sociale et politique ${ }^{50}$. L'Inner Circle, principalement identifié par l'analyse de réseau entre les conseils d'administration des grandes firmes, serait dès lors l'instance d'autorité par excellence des élites économiques, autant que leur porte-parole privilégié. Nous laissons pour l'heure la question épineuse d'une quelconque représentation politique au niveau européen, d'autant plus volontiers qu'une telle représentation unifiée des dirigeants de grandes entreprises en Europe peine déjà à émerger dans les instances consacrées ${ }^{51}$. On peut, en revanche, s'interroger sur le degré de cohésion de ce petit groupe et vérifier s'ils forment entre eux un cénacle. En toute théorie, ils peuvent très bien participer à mettre en relation des sociétés appartenant à des indices différents sans jamais entrer eux-mêmes en relation. Bien sûr, l'absence de relations objectives au travers des sociétés de notre corpus ne retire rien au fait qu'ils peuvent se fréquenter par ailleurs et ainsi boucler la boucle par-delà le champ de vision qui est le nôtre. Nous savons d'ores et déjà que certains d'entre eux se rencontrent en d'autres lieux, que ce soient des conseils d'administration de sociétés non retenues ou d'organisations comme l'European round table for industrialists (ERT). Pourtant, ce serait déjà un signe fort de cohésion transnationale que ces acteurs soient tous interconnectés par le simple jeu des coappartenances aux organes de direction des sociétés retenues.

De fait, la quasi-totalité des interlockers transnationaux forme un seul et même réseau (Graphe 2) d'où est seulement exclue la néerlandaise Karen de Segundo ${ }^{52}$. Le réseau, sans être très dense $(8 \%)$, présente un maillage assez

48. Nollert M., op. cit.

49. Op.cit.

50. Useem M., The inner circle, New York, Oxford University Press, 1984.

51. Pageault A., op. cit.

52. Karen de Segundo a effectué toute sa carrière à la Shell dont elle devint en 1998 directrice 
serré (diamètre $=7$ ). Ainsi, loin d'être un espace fragmenté, le milieu européen des affaires repose sur un réseau d'interconnaissance relativement dense et, surtout, connexe. Il y a loin cependant à y voir une action concertée ou l'expression d'une volonté d'agir collectivement. Il n'existe pas d'organe suprême de coordination qui veillerait à ce que tous ces acteurs soient pris dans un même réseau de relations, ni même une concertation généralisée des entreprises pour connaitre les effets sur le réseau de la nomination de tel ou tel administrateur. D'ailleurs, le réseau tel que nous l'objectivons est généralement ignoré des intéressés eux-mêmes. Le fait que presque tous les interlockers transnationaux soient interconnectés les uns aux autres relève donc d'un hasard, mais d'un hasard structural, qui révèle l'existence et la force d'un groupe social par-delà les intentions des agents.

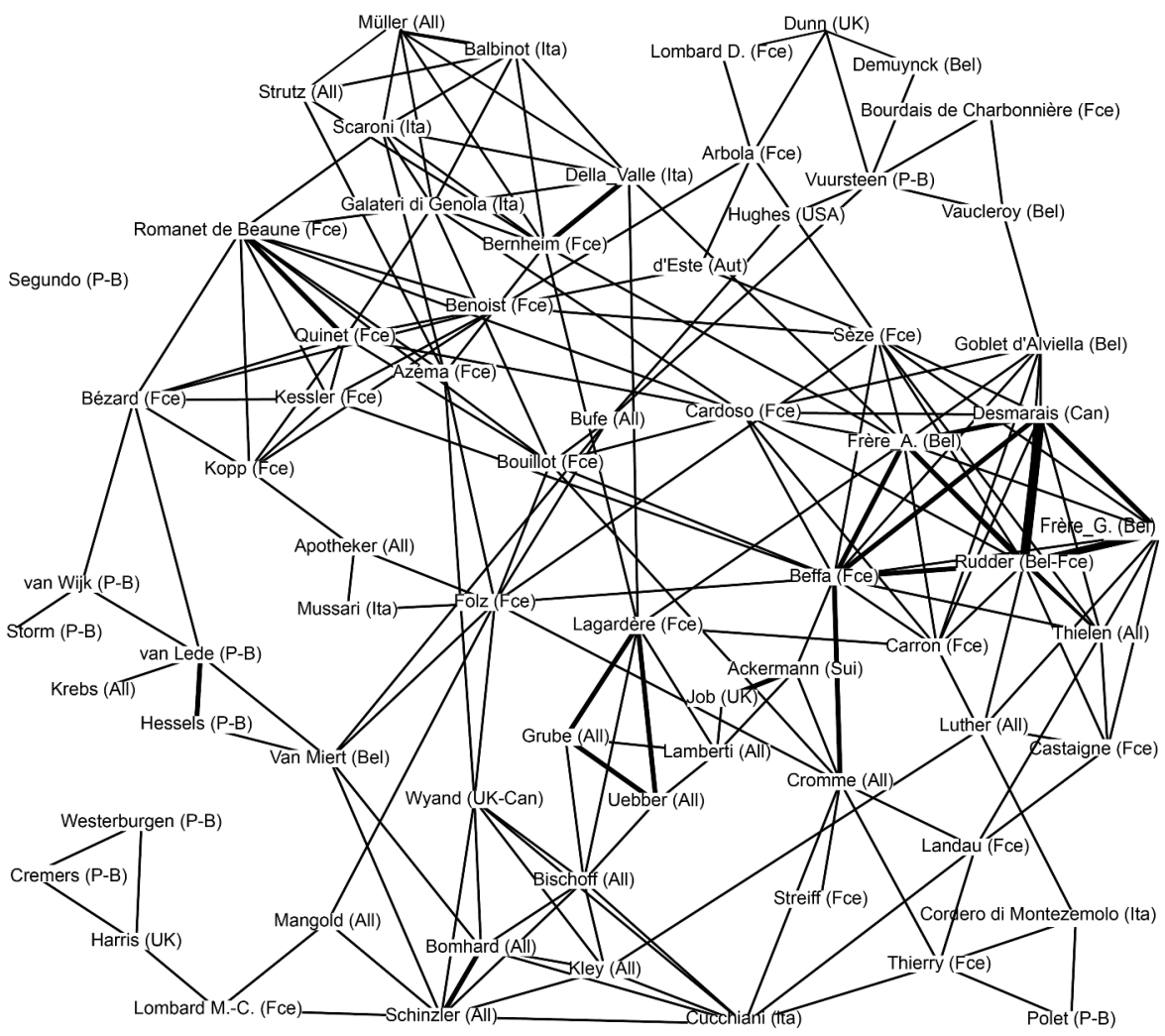

Graphe 2. Réseau des interlockers transnationaux

Légende : All = Allemand $;$ Aut $=$ Autrichien $; B e l=B e l g e ; C a n=$ Canadien $;$ Fce $=$ Français $;$ Ita $=I$ Italien ; $P-B=$ Néerlandais $;$ Sui $=$ Suisse $; U K=$ Britannique $; U S A=$ Américain

générale (CEO) de l'une des quatre principales branches (Gas E Power). Son ascension au sein de la Shell fut brutalement interrompue, en 2000, par un accident de ski qui la conduisit à prendre des responsabilités moins importantes. Elle quitte la société en 2005 tout en continuant de siéger dans de nombreuses entreprises européennes, dont en 2008 l'assureur néerlandais Aegon et l'énergéticien allemand E.ON. 


\section{Quelques propriétés sociales des acteurs-clef}

Sans surprise, les 70 interlockers transnationaux sont très majoritairement des hommes (65 sur 70) présentant une moyenne d'âge plutôt élevée (59,6 ans). Ils sont presque tous européens, mais ne proviennent pas nécessairement des cinq pays privilégiés par l'étude (Tableau 1). Une proportion non négligeable $(11 \%)$ de dirigeants étrangers à la zone euro contribue à faire exister le réseau, venant attester de son caractère global et non strictement transfrontalier. Encore s'agit-il d'une globalité bien délimitée qui s'arrête à l'Europe de l'ouest et à l'Amérique du nord, c'est-à-dire à un espace atlantique des affaires identifié depuis longtemps ${ }^{53}$. Autre enseignement, il n'y a pas de corrélation significative entre la nationalité des interlockers transnationaux et la composi-

\begin{tabular}{|l|c|} 
Nationalité & Individus \\
\hline Allemagne, RFA & 16 \\
Autriche & 1 \\
Belgique & 6 \\
Belgique France & 1 \\
Canada & 1 \\
États-Unis d'Amérique & 1 \\
France & 23 \\
Italie & 7 \\
Pays-Bas & 9 \\
Royaume-Uni & 3 \\
Royaume-Uni Canada & 1 \\
Suisse & 1 \\
Total & $\mathbf{7 0}$ \\
\hline
\end{tabular}

\section{Tableau 1. Nationalité des} acteurs clefs tion des indices boursiers. Ainsi a-t-on autant d'Italiens que de Belges alors que le MIB rassemble 40 firmes et le BEL moitié moins. Enfin, Français et Allemands dominent nettement. Toutes les nationalités ne participent pas de la même manière à la mise en réseau. L'intégration européenne est hétérogène.

Une autre dimension qui vient souligner l'hétérogénéité de ce cénacle transnational est l'origine scolaire de ses membres. Certes, nos acteurs-clef ont presque tous suivi des études supérieures, sauf quatre, généralement en économie (36), en droit et science politique (27) ou encore en ingénierie et sciences (10), combinant souvent les unes avec les autres, mais au-delà de ces proximités disciplinaires, les modèles nationaux, voire impériaux, de production des élites reprennent très vite leur droit. Ainsi, peu nombreux sont ceux qui ont suivi un cursus à l'étranger (16/70). Tous les autres contribuent, par leur parcours scolaire, à souligner la force des tropismes nationaux. Le titre de docteur, véritable sésame outreRhin, est presque exclusivement porté par les dirigeants allemands (9/16), tout juste accompagnés du Suisse Josef Ackermann - est-il utile de préciser qu’il vient de la Suisse alémanique et qu'il acheva sa brillante carrière à la tête de la Deutsche Bank ? De même, le parcours scolaire des Français est marqué par le passage en grande école (17/23), en particulier Sciences-Po (7), l'ENA (5), Polytechnique (4), HEC (3), ainsi que, à la sortie, par l'intégration des corps de la haute fonction publique (8/23), en particulier l'inspection des Finances

53. Pour un historique des travaux sur ce sujet, voir la revue de littérature développée dans Van Der Pijl K., Holman O., Raviv O., "The resurgence of German capital in Europe. EU integration and the restructuring of Atlantic networks of interlocking directorates after 1991", Review of International Political Economy, 18/3, 2011, pp. 384-408. 
et le corps des Mines (4/8). Parmi les trois dirigeants britanniques, deux sont diplômés d'Oxford. Le dirigeant américain est diplômé d'Harvard. Pour les autres pays, l'élitisme scolaire est moins évident, notamment parce que comme aux Pays-Bas, il est moins institutionnalisé. Certains établissements ressortent néanmoins : l’Université de Leiden aux Pays-Bas (4/9), les universités de Bocconi et de Gènes en Italie (4/7). Si ces observations ne valent que pour le groupe étudié et ne sont guère généralisables en raison de la faiblesse de l'effectif, elles sont néanmoins en parfaite cohérence avec ce qu'on sait de la formation des élites économiques en Europe ${ }^{54}$. Les cursus à l'étranger concernent une minorité de dirigeants (16) et se concentrent inégalement sur quatre pays : les États-Unis (13), la France (4), la Suisse (3) et le Royaume-Uni (1). Les trois dirigeants qui ne sont pas passés par les États-Unis sont venus en France, en particulier à l'INSEAD (2). Aux États-Unis, les établissements les plus fréquentés sont Harvard et Columbia (3 fois chacun), suivis de Stanford et Wharton (2 fois chacun). Cette surreprésentation des États-Unis dans les cursus étrangers rappelle la force d'attraction du cœur de l'empire dans la production des élites internationales.

\section{Les parcours professionnels des interlockers transnationaux}

Pour chacun des 70 interlockers transnationaux, nous reconstituons la trajectoire professionnelle, à compter de la fin des études, sur la base de l'occupation d'une fonction donnée au sein d'une institution donnée ${ }^{55}$. Une fonction est une attribution dévolue à un individu pendant un certain nombre d'années au sein d'une institution, par exemple pour une entreprise : employé, manager, top manager (membre de comité exécutif), directeur général, administrateur, président du conseil d'administration. Une institution peut être une entreprise, une administration d'État, un parti politique ou tout type d'association.

À fin 2008, la majorité des acteurs-clefs (37/70) sont présidents ou directeurs général d'une grande entreprise. Les autres sont soit de hauts cadres dirigeants (14/70), soit des « administrateurs de société » (14/70), c'est-à-dire souvent eux-mêmes d'anciens dirigeants de grands groupes qui pour cause de départ à la retraite ou accident de parcours n'occupent plus de fonctions exécutives mais conservent un certain nombre de mandats d'administrateur.

54. Joly H. (ed.), La formation des élites en France et en Allemagne, Paris-Cergy, CIRAC, 2005 ; Sabbagh, D., Van Zanten A., «Diversité et formation des élites : France-USA », Sociétés contemporaines, 79/3, 2010, pp. 5-17 ; Davoine E., Ravasi C., "The relative stability of national top management career profiles in the age of globalization. A comparison of France, Germany, Great Britain and Switzerland”, European Management Journal, 31, 2013, pp. 152163.

55. Seule la trajectoire principale est ici prise en compte et non les affiliations et fonctions jugées secondaires, qui si elles seraient intéressantes à intégrer à l'analyse, posent un certain nombre de problèmes statistiques, notamment dans une perspective diachronique, qui n'ont pas encore été résolus. 
Enfin, on trouve deux présidents d'entreprise moyenne, deux hauts fonctionnaires (dont le directeur général de la Caisse des dépôts et consignations) et un associé gérant d'une banque suisse, Lorenz d'Este, héritier des Habsbourg et beau-frère de Philippe II de Belgique. Une majorité relative exerce son activité principale dans la finance (24/70), suivie de près par l'industrie (23/70), les autres secteurs (monde politico-administratif et culture-éducation-recherche) arrivent loin derrière.

La très grande majorité des interlockers transnationaux a fait toute sa carrière au sein de grandes organisations, qu'elles soient liées à l'État ou non. Rappelons que l'idéal type wébérien de la bureaucratie ne fait pas de différence entre l'administration « publique » ou « privée ». Il serait d'ailleurs plus correct de parler de bureaucratie d'État et de bureaucratie Corporate. Le tableau 2 agrège le nombre d'années cumulées par type d'organisations traversées : grande entreprise (plus de mille salariés), autre entreprise (estimée à moins de mille salariés y compris les holdings familiales), monde politicoadministratif (administration publique et mandat politique), recherche-enseignement-culture, absence d'affiliation principale (administrateurs de sociétés). Les carrières en grande entreprise arrivent très largement en tête (2 054 années cumulées). Si on y ajoute celles associées au monde politico-administratif (274

\begin{tabular}{|l|c|} 
Types d'organisations & $\begin{array}{c}\text { Nombre } \\
\text { d'années } \\
\text { cumulées }\end{array}$ \\
\hline Grande entreprise & 2054 \\
Autre entreprise & 450 \\
Politico-administratif & 274 \\
Sans affiliation & 121 \\
Éducation/Recherche/Culture & 63 \\
\hline Total & $\mathbf{2 9 6 2}$ \\
\hline
\end{tabular}

Tableau 2. Types d'organisations traversées par les 70 interlockers transnationaux

années cumulées), c'est près de $85 \%$ du temps de carrière cumulé qui s'est effectué dans l'administration. Sous cet aspect, l'élite transnationale des affaires que nous observons forme plus un cénacle de bureaucrates au sens où l'entendait Weber qu'un faisceau d'entrepreneurs.

La grande majorité des dirigeants a commencé sa carrière comme employé ou cadre subalterne de grandes firmes multinationales ou de l'administration publique avant de gravir les échelons de la hiérarchie administrative. Ainsi, le Français Jean-Louis Beffa, polytechnicien, sorti ingénieur du corps des Mines, débute sa carrière, en 1967, au ministère de l'industrie. En 1974, il rejoint Saint-Gobain comme directeur du Plan avant de s'imposer progressivement comme le successeur désigné de Roger Fauroux, auquel il succède en 1986. En 2007, il quitte la direction exécutive de l'entreprise mais conserve la présidence du conseil d'administration jusqu'en 2010. En 2008, outre Saint-Gobain, il siégeait aux conseils d'administrations du Groupe Bruxelles Lambert, BNPParibas, GDF-Suez et Siemens. Autre trajectoire emblématique celle de l'ita- 
lien Gabriele Galateri di Genola. Licencié en droit (La Sapienza - Rome) et titulaire d'un MBA de l'université de Columbia, il commence sa carrière en 1971 chez Banca di Roma comme cadre supérieur, avant de rejoindre Saint Gobain comme directeur financier pour l'Italie (1974-1976). En 1977, il prend la responsabilité des opérations financières de Fiat aux Amériques avant de devenir directeur financier du groupe. De 1986 à 2002, il dirige les boldings financières de la famille Agnelli puis prend brièvement la tête de Fiat. À compter de 2003, il devient l'une des figures phares du capitalisme italien, présidant successivement les conseils de Mediocanca (2003-2007), de Telecom Italia (2007-2011) et depuis 2011 celui de Generali. En 2008, outre Telecom Italia, il siégeait aux conseils d'Accor et Generali. L'ensemble des trajectoires observées suivent une dynamique ascendante tant du point de vue des fonctions exercées (cadre, cadre supérieur, cadre dirigeant, chef de la direction exécutive ou président du conseil) que de la taille de l'entreprise (filiale vers société mère). Ce type de parcours révèle un véritable cursus bureaucratique propre au monde des grandes entreprises et que l'on peut qualifier de « corporatique » 56 pour le distinguer d'autres types de trajectoires bureaucratiques propres à l'administration d'État. L'un des faits frappants est la fréquence de ce type de carrière «corporatique » au-delà des diversités nationales soulignées par ailleurs.

Certes, ces parcours sont ceux des dominants, de dirigeants qui ont passé avec succès les différentes étapes du cursus honorum de la grande entreprise, depuis l'obtention des titres scolaires jusqu'aux plus hautes fonctions et même au-delà en devenant des membres de l'élite transnationale des affaires. Ils représentent donc des modèles de réussite professionnelle.

Il existe bien entendu des exceptions à ce modèle « corporatique » 57 , tels ces dirigeants, héritant d'une petite affaire familiale, qui ont su la transformer en multinationale (Albert Frère avec Frère-Bourgeois ou Diego Della Valle avec Tod's), ou le parcours totalement atypique de Léo Apotheker, hésitant une bonne partie de sa carrière entre la grande et la petite entreprise, le carriérisme « corporatique » et l'aventure « entrepreneuriale ». De la même manière, la carrière strictement politique d'un Karel van Miert 58 dénote singulièrement

56. Le recours au suffixe «-iste » susciterait sans doute plus de confusions encore que notre néologisme.

57. Par «corporatique » nous entendons l'organisation bureaucratique propre au monde des grandes entreprises.

58. Il débute sa carrière comme membre du cabinet du commissaire européen Sicco Mansholt (1968-1969). Il enseigne quelque temps le droit international à l'Université libre de Bruxelles, avant de retourner auprès de la Commission européenne dans le cabinet d'Henri Simonet (1973-1975). Fortement investi dans les partis socialistes belges et flamands, il entre au cabinet du ministre de l'économie Willy Claes (1977). En 1979, il devient député européen, puis député belge en 1985. En 1989, il est nommé commissaire européen en charge des transports, du crédit et de l'investissement, puis à compter de 1993 en charge de la concurrence. De 2000 à 2003, il préside l'université de Nyenrode. Depuis 2003, il était à titre principal enseignant dans cette même université tout en cumulant un nombre impressionnant de mandats d'administrateur. Il est décédé accidentellement en 2009. 
même si elle ne jure pas. Mais dans tous les cas, que ce soit par la transformation de la petite entreprise en grande ou par un ralliement plus ou moins tardif, le modèle «corporatique » finit par s'imposer à tous, ne serait-ce que par les fonctions qu'ils exercent à la tête des grandes entreprises (ils sont, par définition, au moins deux fois dirigeants d'un grand groupe). Même si la direction des grandes entreprises fonctionne sur une base collégiale tant à l'intérieur de l'entreprise (conseil d'administration - comité exécutif) qu'à l'extérieur (réseaux d'administrateurs), il s'agit d'une collégialité inscrite dans un univers fondamentalement bureaucratique ${ }^{59}$.

\section{Analyse séquentielle des carrières professionnelles}

Toutefois, la fréquence des parcours « corporatiques » ne nous renseigne guère sur l'orientation financière ou industrielle de la carrière. À cette fin, nous avons distribué les différentes institutions présentes dans notre corpus en fonction de cinq « macro-secteurs » recoupant partiellement les types d'organisations : finance ${ }^{60}$, industrie ${ }^{61}$, monde politico-administratif, Éducationrecherche-culture et, pour ceux qui n'avaient pas d'affiliation principale, la catégorie ad hoc «d'administrateur de sociétés ». Afin de raffiner quelque peu notre étude nous avons distingué parmi les trajectoires dans l'industrie et le monde politico-administratif les fonctions financières (directeur financier, haut fonctionnaire du Trésor). C'est en rapport avec ce nouveau découpage que nous avons établi les séquences individuelles d'activité principale.

L'analyse de séquences consiste à analyser des populations de séquences ${ }^{62}$ afin de les décrire statistiquement et graphiquement, de les classifier, de les fouiller, de repérer des séquences typiques et les facteurs qui les expliquent. Cette méthode statistique prend l'avantage sur les méthodes concurrentes d'analyse des données longitudinales grâce à la prise en compte de trois dimensions du

59. Max Weber évoquait déjà la possibilité d'une certaine collégialité dans les organisations bureaucratiques, même si pour lui elle était historiquement située et transitoire. Sur les formes de collégialité au sein des organisations bureaucratiques et le caractère heuristique de l'analyse de réseaux pour en saisir les formes de discipline qui s'y exercent voir Lazega E., The Collegial Phenomenon, The Social Mechanisms of Cooperation among Peers in a Corporate Law Partnership, Oxford, Oxford University Press, 2001.

60. Nous entendons par finance les entreprises exerçant le métier de la banque, de l'assurance, de l'investissement ainsi que l'ensemble des services financiers dédiés tels que les chambres de compensations, les marchés financiers etc... Plus généralement l'ensemble des professionnels dont le métier premier est lié à l'émission et la circulation de la monnaie. Voir Dudouet F.-X., Grémont E., Vion A., « La “centralité des banques”... », op. cit.

61. Nous mettons dans industrie tout ce qui n'est pas finance, la distinction entre service et industrie étant souvent très problématique pour les groupes étudiés. En revanche, la distinction entre les entreprises financières et non financières est au cœur des débats menées par les interlocking directorate studies sur le pouvoir de la finance. Voir Scott J., op. cit.

62. Une séquence est une suite d'états ou d'événements définis dans un alphabet judicieusement créé. Voir Blanchard P., Bühlmann F., Gauthier J.-A. (eds.), Advances in sequence analysis. Methods, theories and applications, London, Springer, 2014. 
temps (durée dans un état donné, ordre des états), la saisie des séquences dans leur entièreté et non par un découpage du temps a priori, l'absence d'hypothèses statistiques contraignantes sur la structure des données et une meilleure prise en charge d'une variété de tailles de populations, de longueurs de séquences et de données manquantes ${ }^{63}$. Elle a été importée de l'informatique et la génétique vers la sociologie du travail et de la famille ${ }^{64}$, avant de toucher la sociologie économique 65 .

Le graphe 3 présente les trajectoires professionnelles des 70 interlockers transnationaux sous forme de séquences par macro-secteur et fonctions financières : industrie (gris foncé), industrie avec fonction financière (gris foncé hachuré blanc), finance (gris moyen), politique et administration (gris souris), politique et administration avec fonction financière (gris souris hachuré blanc), recherche-formation-culture (gris clair). Les purs administrateurs de sociétés sont en gris clair hachuré blanc. Chaque ligne représente un individu, avec le patronyme à gauche. Les trajectoires ont été ordonnées suivant leur similitude pour faciliter la lecture. On remarque en haut et en bas des trajectoires assez homogènes largement dominées par un secteur particulier : la finance (gris moyen) en haut et l'industrie (gris foncé) en bas. Au centre se regroupent des trajectoires plus hétérogènes et composées de secteurs moins dominants. Les carrières se font souvent au sein d'un même macro-secteur (parfois au sein d'une même entreprise), indiquant une mobilité assez faible entre le monde de la finance et le reste de l'économie.

Cette représentation permet de distinguer deux grands types de carrières bien distincts, l'un presque exclusivement industriel, l'autre principalement financier. Ces deux modèles rassemblent à eux seuls $66 \%$ de l'effectif avec une nette prime pour l'industrie (28/70), la finance étant nettement en retrait (18/70). Ces chiffres doivent être rapportés à ceux observés en 2008, c'est-àdire à l'extrémité droite du graphe. Financiers et industriels sont alors au coude à coude (24/23) avec une légère prime pour les premiers. Cette différence entre trajectoires et situations finales peut recevoir deux interprétations.

63. Abbott A., "Sequence Analysis: New Methods for Old Ideas", Annual Review of Sociology, 21, 1995, pp. 93-113 ; Blanchard P., "Sequence analysis for political science", Working papers of the Committee on concepts and methods, International political science association, 2011: http://www.concepts-methods.org/WorkingPapers/PDF/1082 (Page consultée le 24 juillet 2015) ; Blanchard P., Bühlmann F., Gauthier J.-A. (eds.), op. cit.

64. Blanchard P., " "Validity, falsifiability, parsimony, consistency, precision, and so on". Les vicissitudes de l'innovation méthodologique ", in Jouvenet M., Demazière D. (eds.), La sociologie d'Andrew Abbott, Paris, EHESS, à paraître.

65. Blair-Loy M., "Career patterns of executive women in finance. An optimal matching analysis", American journal of sociology, 104, 1999, pp. 1346-97 ; Bühlmann F., "The corrosion of career? Occupational trajectories of business economists and engineers in Switzerland", European Sociological Review, 24/5, 2008, pp. 601-616 ; Lemercier C., " Les carrières des membres des institutions consulaires parisiennes au XIXe siècle ", Histoire et mesure, XX/1-2, 2005, pp. 59-95. 
L'une est biographique : les financiers restent fidèles à leur orientation d'origine, tandis que les industriels tendent à changer de secteur ou à prendre leur retraite 66 ; l'autre est historique : la finance domine de plus en plus l'élite corporatique transnationale.

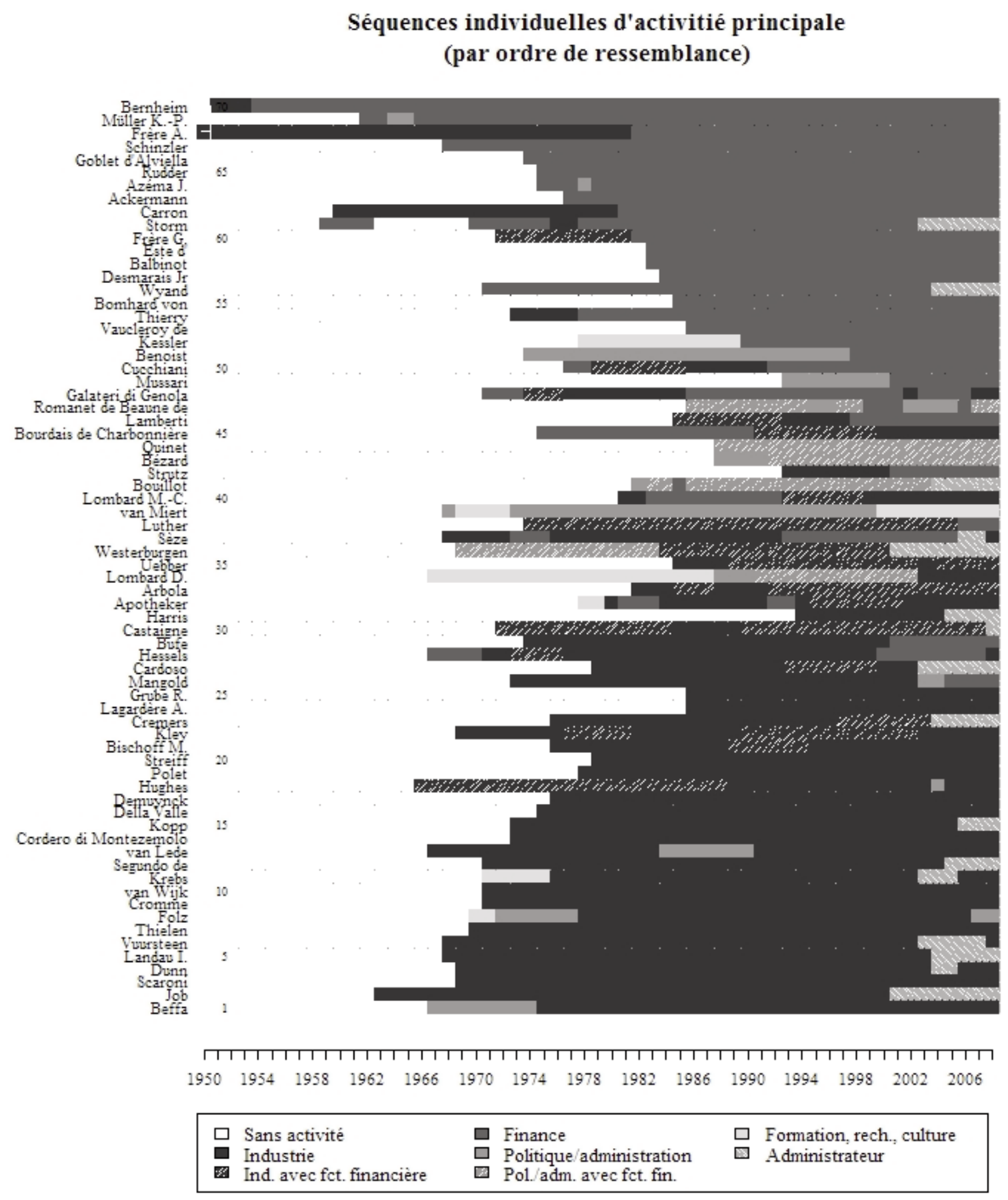

Graphe 3. Trajectoires des interlockers transnationaux $(\mathrm{N}=70)$

66. Même si le doyen du corpus, Antoine Bernheim, 84 ans en 2008, est un ancien associé gérant de la Banque Lazard et le président en exercice de Generali, le nombre de « retraités " parmi les industriels est trois fois plus nombreux que parmi les financiers. 
Afin d'approfondir l'analyse de ces trajectoires, nous les analysons ensuite à l'aide de l'algorithme d'appariement optimal, ou optimal matching ${ }^{67}$. L'indice de dissimilarité des paires de séquences est calculé sur la base d'une matrice de coûts respectant deux principes simples : les secteurs sont tous identiquement distincts les uns des autres; les personnes occupant une fonction financière hors du secteur financier, dans l'industrie par exemple, sont proches de leur secteur mais aussi relativement proches de la finance. Nous obtenons une matrice compilant les dissimilarités associées à toutes les paires de séquences. Nous appliquons ensuite une classification ascendante hiérarchique, qui regroupe de proche en proche les profils de distance les plus ressemblants jusqu'à atteindre un nombre de classes raisonnable. Cette ressemblance est basée sur l'algorithme de Ward, qui a l'avantage de déboucher sur des classes de taille similaire et néanmoins homogènes (graphe 4). Le tableau 3 met en exergue, pour chaque classe, trois types de propriétés sociales : la nationalité ; la date de naissance, qui marque l'inscription dans une génération de dirigeants; et le nombre de mandats. Nous pouvons à présent décrire et interpréter la typologie.

La première classe de carrières correspond aux carrières longues au sein d'entreprises financières, avec moins de cinq fonctions et moins de trois institutions traversées. Les passages par l'industrie sont généralement brefs, et plutôt en début de carrière ${ }^{68}$. On a ici une classe de purs financiers tels le Suisse Josef Ackermann, qui débute sa carrière au Crédit Suisse en 1977 avant de rejoindre la Deutsche Bank en 1996, dont il prendra la tête en 2002 ; Sergio Balbinot (Italien), qui a fait toute sa carrière chez Generali ; Klaus-Peter Müller (Allemand), entré en apprentissage dans la banque Friedrich Simon en 1962, qui rejoint la Commerz Bank en 1966 après son service militaire pour en devenir le président exécutif en 2001, jusqu'à son rachat par Allianz en 2008, consécutivement à sa quasi-faillite. Les interlockers transnationaux de cette classe sont des dirigeants aux statuts divers, issus de grandes institutions financières (Deutshe Bank, Generali, Crédit Agricole, Aviva, Commerzbank, etc.) et fortement impliqués dans le financement des entreprises et des bourses à l'échelle européenne, voir mondiale. On retrouve dans cette classe les reliquats du capitalisme familial converti à la finance. Ainsi en est-il des familles Boël, Desmarais et Frère dont les conglomérats industriels sont devenus financiers dans les années 1980. On notera aussi que dans cette classe, la proportion de non ressortissants de la zone euro est plus élevée (environ un quart). Outre Paul Desmarais Jr. (Canada), on y trouve Anthony Wyand,

67. Pour une explication détaillée de l'appariement optimal, voir Blanchard P., "Sequence Analysis...", op. cit.

68. Notons que nous classons le début de carrière de René Carron dans l'industrie en assimilant l'élevage de vaches laitières à une activité industrielle. René Carron est en effet un exploitant agricole savoyard qui a gravi les échelons du Crédit agricole jusqu'à en devenir président de la Fédération nationale des caisses de crédit agricole et à introduire le Crédit Agricole SA en bourse. 
citoyen canadien et britannique, qui a accompagné les restructurations de l'assureur britannique CGU jusqu'au groupe Aviva, et qui administre en 2008 deux banques de premier plan de la zone euro : Société Générale et Unicredito, de même que le Suisse Josef Ackermann, président de la Deutsche Bank et qui a représenté en 2011 les créanciers privés de la Grèce sur le dossier de la remise de créance des banques. Notons enfin, que cette classe n'est composée que d'hommes, dont la grande majorité a plus de 50 ans en 2008.

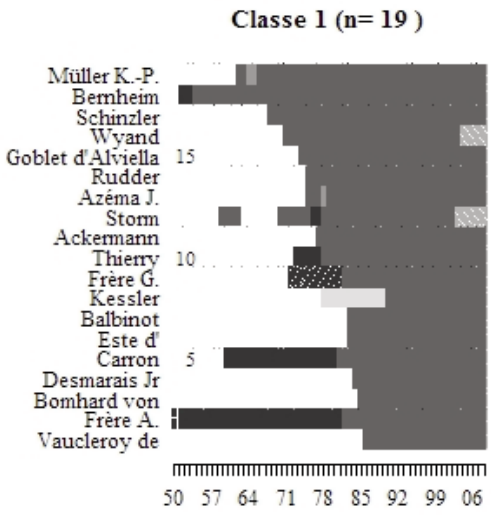

Classe $3(n=14)$

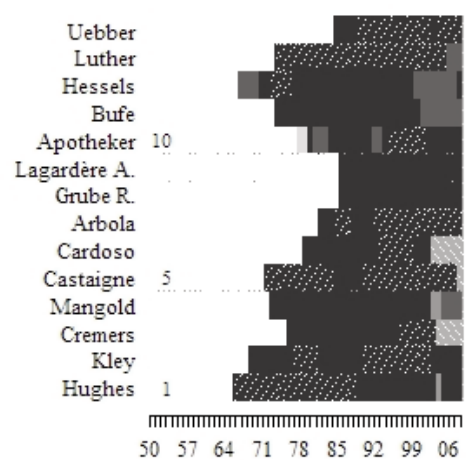

Classe $5(n=8)$

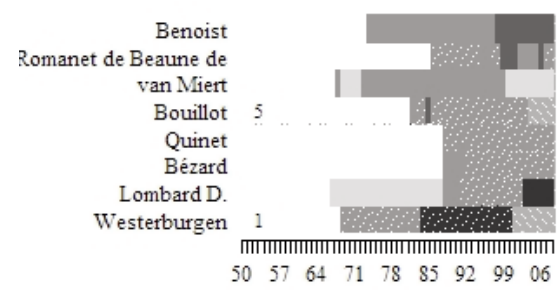

Classe $2(n=20)$

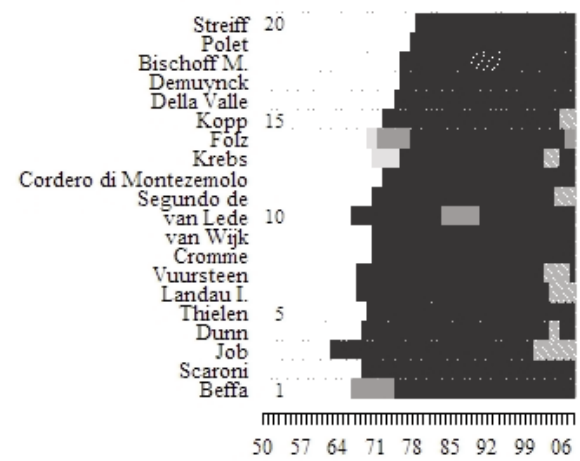

Classe $4(n=9)$

Galateri di Genola

Cucchiani

Mussari

Bourdais de Charbonnière

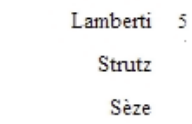

Lombard M.-C.

Harris 1

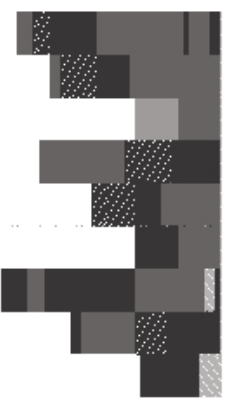

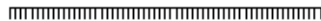
$\begin{array}{lllllllll}50 & 57 & 64 & 71 & 78 & 85 & 92 & 99 & 06\end{array}$

\begin{tabular}{|l}
\hline Sans activité \\
$\square$ Industrie \\
$\square$ Ind. avec fct. financière \\
$\square$ Finance \\
$\square$ Politique/administration \\
$\square$ Pol./adm. avec fct. fin. \\
$\square$ Formation, rech., culture \\
$\square$ Administrateur de société
\end{tabular}

Graphe 4. Typologie des séquences de trajectoire par macro-secteur 


\begin{tabular}{lcccccc}
\hline & $\mathbf{1}$ & $\mathbf{2}$ & $\mathbf{3}$ & $\mathbf{4}$ & $\mathbf{5}$ & $\mathbf{N = 7 0}$ \\
\hline Nationalité & & & & & & \\
\hline Allemagne, RFA & 16 & 20 & 50 & 22 & 0 & 23 \\
\hline Autriche & 5 & 0 & 0 & 0 & 0 & 1 \\
\hline Belgique & 21 & 5 & 0 & 0 & 12 & 9 \\
\hline Canada & 5 & 0 & 0 & 0 & 0 & 1 \\
\hline États-Unis & 0 & 0 & 7 & 0 & 0 & 1 \\
\hline France & 26 & 25 & 29 & 33 & 75 & 33 \\
\hline France et Belgique & 5 & 0 & 0 & 0 & 0 & 1 \\
\hline Italie & 5 & 15 & 0 & 33 & 0 & 10 \\
\hline Pays-Bas & 5 & 25 & 14 & 0 & 12 & 13 \\
\hline Royaume-Uni & 0 & 10 & 0 & 11 & 0 & 4 \\
\hline Royaume-Uni et Canada & 5 & 0 & 0 & 0 & 0 & 1 \\
\hline Suisse & 5 & 0 & 0 & 0 & 0 & 1 \\
\hline
\end{tabular}

Naissance

\begin{tabular}{lcccccc}
\hline Années 1920 & 11 & 0 & 0 & 0 & 0 & 3 \\
\hline Années 1930 & 0 & 0 & 0 & 11 & 0 & 1 \\
\hline Années 1940 & 47 & 80 & 57 & 22 & 62 & 57 \\
\hline Années 1950 & 37 & 20 & 36 & 33 & 0 & 27 \\
\hline Années 1960 & 5 & 0 & 7 & 33 & 38 & 11 \\
\hline Âge moyen en 2008 & 61,3 & 62,3 & 58,9 & 53,8 & 57,2 & $\mathbf{5 9 , 7}$ \\
\hline
\end{tabular}

Carrière

\begin{tabular}{lcccccc}
\hline Nb moyen de fonctions occupées & 4,7 & 6 & 6,1 & 6 & 9,1 & 6,0 \\
\hline Nb moyen d'institutions traversées & 2,8 & 3,7 & 3,5 & 4 & 4,2 & 3,5 \\
\hline & & & & & & \\
\hline Total & $\mathbf{1 0 0}$ & $\mathbf{1 0 0}$ & $\mathbf{1 0 0}$ & $\mathbf{1 0 0}$ & $\mathbf{1 0 0}$ & $\mathbf{1 0 0}$ \\
\hline
\end{tabular}

Tableau 3. Propriétés des types de séquences

Pourcentage de chaque modalité dans chaque classe

La deuxième classe correspond aux industriels purs, à ceux qui ont fait la quasi-totalité de leur carrière dans l'industrie, incarnant une forme de consécration de la mobilité ascendante dans ce secteur. On y trouve Gerhard Cromme, le président de ThyssenKrupps et de Siemens, principal animateur avec son ami Jean-Louis Beffa (Saint-Gobain) des tentatives de relance de coopérations franco-allemandes durant les années 200069 ; Diego della Valle, pré-

69. La relation d'amitié entre les deux hommes, qui date certainement du passage de Cromme chez Saint-Gobain (1971-1986), a longtemps constitué le pilier incontournable des projets franco-allemands en matière d'innovation industrielle ces dernières années. Selon un diplomate français, aucun projet entre les deux rives du Rhin ne pouvait à cette période aller à son terme sans l'appui plus ou moins direct de l'un des deux hommes. La retraite de Jean-Louis 
sident de Tod's ; Rüdiger Grube, qui a fait toute sa carrière chez Daimler avant de prendre la tête de la Deutsche Bahn en 2009 ; Luca Cordero di Montezomolo, président emblématique de Ferrari puis de Fiat à partir de 2004 et ami intime de Diego Della Valle ; Leo van Wijk, ancien président de KLM et artisan de la fusion avec Air-France, etc. Trois d'entre eux sont en 2008 membres de l'ERT : Jean-Louis Beffa, Gerhard Cromme et Paolo Scaroni. Les cinq pays de notre échantillon de base sont représentés. L'inscription générationnelle des dirigeants de cette classe dans les années 40 et 50 est également forte. Il serait cependant hâtif de parler de « vieille garde » des industriels, si l'on conçoit ce terme comme la direction d'activités industrielles en déclin toutes ne le sont pas, loin s'en faut.

La troisième classe se compose d'industriels un peu plus jeunes que les précédents, avec néanmoins des profils financiers plus marqués. La plupart d'entre eux, bien qu'ayant une carrière inscrite dans l'industrie, a exercé des fonctions financières ou a bifurqué vers la finance en fin de carrière. Un petit nombre garde cependant un profil de pur industriel, mais les dirigeants en question sont encore suffisamment jeunes pour que leur trajectoire puisse prendre un tour «financier». Louis R. Hughes (Américain) a ainsi effectué la quasi-totalité de sa carrière chez General Motors, d'abord au sein du Treasurer Department pendant près de 20 ans (1966-1985) puis comme VicePresident Finance de GM Canada (1985-1987) et de GM Europe (1987-1989). Ce n'est que vers la fin de sa carrière, en prenant la direction d'Opel (filiale allemande de GM), puis en devenant Vice-President de General Motors Corporation, qu'il quittera des fonctions strictement financières, avant de les retrouver partiellement en 2005 en devenant premier actionnaire et dirigeant de la firme de sécurité informatique InZero. On peut aussi citer Uwe-Ernest Bufe (Allemand) qui, après avoir effectué toute sa carrière chez Degussa (chimie), est recruté après son départ à la retraite par UBS, tout en cumulant les mandats d'administrateur dans les plus grandes entreprises européennes de chimie (Air Liquide, Altana, AkzoNobel, K+S, Solvay ou encore Umicore). Aldo Cardoso, de nationalité française, a effectué toute sa carrière chez Andersen consulting dont il fut un temps responsable de l'audit en France. Nommé président d'Andersen France en 1998, il devient le président exécutif du groupe au niveau mondial en 2002, au moment de la mise en liquidation de la société suite à l'affaire ENRON. Il est depuis « administrateur de sociétés ", notamment auprès d'Accor, AXA Investment Managers, Bureau Veritas, Gaz de France puis GDF-Suez, Gecina, Imerys, Mobistar (filiale de France Telecom), Orange et Rhodia. La classe 3 regroupe donc des industriels au profil financier marqué ou de purs industriels susceptibles de recevoir un marquage financier dans la suite de leur carrière.

Beffa a d'ailleurs fortement compliqué les choses par la suite. Entretien avec Jean-François Boittin, Ministre Conseiller à l'Ambassade de France à Washington, ancien Ministre conseiller pour les affaires économiques auprès de l'ambassade de France en Allemagne. Entretien effectué le 14 novembre 2013 à Washington. 
La quatrième classe regroupe des dirigeants à la trajectoire caractérisée par une bifurcation sectorielle très prononcée qui se situe plutôt en milieu de carrière. Le passage se fait indistinctement de l'industrie vers la finance ou inversement, un cas du monde politico-administratif vers la finance et un autre de l'industrie vers « administrateur de sociétés ». Eric Bourdais de Charbonnière est devenu directeur financier puis président du conseil de surveillance de Michelin après avoir dirigé les activités de JP Morgan en France et en Europe. Amaury Daniel de Sèze a connu un parcours exactement inverse en débutant sa carrière dans l'industrie, notamment chez Volvo, avant de rejoindre Paribas au début des années 1990 et de prendre la tête de son pôle d'investissement Paribas affaires industrielles, devenu PAI Partners en 2003. Trois d'entre eux ont commencé dans le conseil : Enrico Cucchiani et Mary Harris chez Mc Kinsey, Eric Strutz au Boston Consulting Group. Giuseppe Mussari a, quant à lui, bénéficié des vertus du localisme en passant de la chambre criminelle de Sienne où il exerçait comme magistrat à la Banco Monte dei Paschi di Siena. La trajectoire de Gabriele Galateri di Genola est néanmoins plus sophistiquée (cf. supra).

La cinquième classe regroupe des carrières placées sous le sceau de l'État, que ce soit dans l'administration ou la politique. Le passage en entreprise, quand il a lieu, est souvent tardif, intervenant pour trois cas sur quatre dans le dernier tiers de la carrière. Les profils se distribuent donc entre des carrières purement étatiques à l'image d'un Bruno Bézard, inspecteur des Finances, qui n’a jamais quitté Bercy sauf deux ans passés au cabinet du Premier Ministre (Jospin) et un Jos Westerburgen, lui aussi haut fonctionnaire du Trésor néerlandais, mais qui le quitte en milieu de carrière pour rejoindre la direction fiscale d'Unilever, avant de multiplier les mandats d'administrateur dans les années 2000. Pour administratives et politiques que soient ces carrières, elles ne sont guère éloignées des entreprises, la plupart ont exercé des responsabilités financières et même celui qui a eu une carrière exclusivement politique, le Belge Karel van Miert, fut en charge du crédit puis de la concurrence lors de ses mandats de commissaire européen. Ces dirigeants sont donc loin d'incarner une figure du serviteur de l'État détaché du monde des grandes entreprises qui serait regardé de haut, mais représentent au contraire des intermédiaires puissants entre l'univers politico-administratif et celui des grandes entreprises. Encore convient-il préciser que cette classe regroupe avant tout des Français (6/8), ce qui tend à souligner la place particulière qu'occupe si ce n'est l'État en tant que tel, du moins ceux qui en sont issus, dans le capitalisme hexagonal 70. À noter que la moyenne des fonctions occupées dans la carrière est très largement au-dessus des autres classes. Cela s'explique en grande partie par le modèle de carrière de la bureaucratie d'État française où les hauts fonctionnaires sont appelés à changer très régulièrement de poste. Une mobilité que renforce la participation, quasi obligatoire, à des cabinets ministériels.

70. Dudouet F.-X. et al., « Retour sur le champ du pouvoir... », op. cit. 
On trouve avec ces parcours un facteur d'homogénéisation qui jusqu'à présent échappait aux approches en terme de formation ou d'analyses de réseaux qui soulignaient la force des tropismes nationaux ${ }^{71}$. Même si certaines nationalités sont mieux représentées dans certaines classes que d'autres, on ne peut associer telle classe à telle nationalité, si ce n'est pour la dernière, celle du monde politico-administratif où les Français composent trois quarts de l'effectif. Cette classification, si elle fait apparaitre deux grands secteurs d'activité, la finance et l'industrie, souligne aussi la mobilité qui existe entre ces deux sphères. Les classes 3 et 4, près d'un tiers de l'effectif, manifestent cette mobilité, que ce soit au sein de l'industrie par l'exercice de fonctions financières (classe 3) ou en changeant radicalement de secteur en cours de carrière (classe 4). Plus généralement, notre analyse montre la forte prédominance des carrières associées à la finance, que ce soit pour avoir travaillé dans le secteur de la finance ou pour avoir exercé des fonctions financières dans l'industrie ou l'univers politico-administratif. Moins d'un tiers des carrières n'est jamais associé à une institution financière ou une activité financière. Certes, cette prépondérance des profils financiers doit beaucoup aux directeurs financiers de l'industrie et aux fonctionnaires du Trésor, mais cela prouve justement le rôle de pivot de la finance. Si les profils financiers dominent autant parmi les agents qui construisent le milieu des affaires, c'est bien que la finance occupe une fonction systémique et qu'elle incarne un véritable langage commun. Ce constat affine l'analyse de la centralité des financiers dans la zone euro, en montrant que l'attractivité de la compétence financière n'est pas seulement synchronique, comme cela est prouvé sur la base de données réseau en année N. Elle est clairement aussi diachronique, dans la mesure où une activité financière continue de façonner le profil d'un dirigeant, même après qu'elle a cessé. De fait, le passage par une activité financière, plus ou moins longue, est un trait dominant pour toutes les classes sauf la classe 2, qui regroupe les carrières strictement industrielles. Ainsi, le corpus de dirigeants sur la base duquel Nollert 72 construit son inner circle européen (les dirigeants des entreprises industrielles de l'ERT en 1994) représente dans notre étude une classe particulière de dirigeants. Sans être marginale quantitativement, cette classe repose peut-être sur des caractéristiques professionnelles exceptionnelles ou en cours de dévaluation : à savoir une expérience de la conduite de grands projets industriels à caractère multilatéral.

\section{Conclusion}

Revenons sur la contribution spécifique de notre étude à la compréhension du milieu des affaires européen et des élites transnationales. En premier lieu l'analyse de réseau a permis de mettre à jour un espace social transnational sui generis qui se distingue d'autres formes de groupements transnationaux 
de nature plus politique comme l'European round table of industrialists (ERT). En effet, ce qui distingue fondamentalement l'espace social des interlockers transnationaux des membres de l'ERT ${ }^{73}$ est que les seconds sont cooptés par l'ensemble des membres, alors que les premiers le sont uniquement par les conseils d'administrations auxquels ils appartiennent. Or, à moins de supposer une coordination explicite entre les différents conseils pour nommer des administrateurs qui soient tous, ou presque, interconnectés les uns aux autres, force est de constater que la société transnationale ainsi formée est un fait social sui generis. Cela révèle un visage du milieu des affaires transnational beaucoup moins dépendant de la seule figure du grand patron, mais relevant de combinaisons plus subtiles de ressources qui vont du contrôle du capital financier à la maitrise des rouages des institutions européennes, en passant par des types complexes de trajectoires professionnelles. En effet, alors que les analyses en terme de réseaux ou de formation avaient jusqu'à présent souligné la force des tropismes nationaux, l'approche par les trajectoires met en exergue des formes d'homogénéité qui tiennent au caractère bureaucratique des carrières en grandes entreprises. Nous qualifions ces carrières de " corporatiques » pour les distinguer de celles menées dans l'administration d'État. Mais dans les deux cas il s'agit toujours de bureaucratie au sens où l'a définie Weber.

Dans ce registre des carrières, l'analyse séquentielle apporte des éclairages nouveaux sur la structuration de cet espace qu'il était difficile d'obtenir autrement. Elle permet d'affiner considérablement les types de carrières suivies et de mettre en exergue la prédominance des profils associés à la finance. 
Répertoire des interlockers transnationaux Avec leur date de naissance, leur pays d'origine et leurs affiliations aux sociétés du corpus.

Ackermann Josef

1948 Suisse

AEX 25 : Shell

DAX 30 : Deutsche Bank, Siemens

\section{Apotheker Léo}

1953 Allemagne, RFA

CAC 40 : AXA, Schneider

DAX 30 : SAP

\section{Arbola Gérald}

1948 France

CAC 40 : Suez

Environnement

MIB 40 :

STMicroelectronics

\section{Azéma Jean}

1953 France

CAC 40 : Société

Générale, Veolia

MIB 40: Mediobanca

\section{Balbinot Sergio}

1958 Italie

DAX 30 : Commerzbank MIB 40 : Generali

\section{Beffa Jean-Louis}

1941 France

BEL $20: G B L$

CAC 40 : BNP Paribas,

GDF-SUEZ, Saint-Gobain

DAX 30 : Siemens

\section{Benoist Gilles}

1946 France

BEL 20 : Dexia

CAC 40 : Suez

Environnement

Bernheim Antoine
1924 France
CAC $40:$ LVMH
MIB 40 : Alleanza,
Generali, Intesa
Sanpaolo,
Mediobanca
Bézard Bruno
1963 France
BEL 20 : Dexia
CAC 40 : Air France-KLM,
EDF SA

\section{Bischoff Manfred}

1942 Allemagne, RFA

AEX $25: K P N$

DAX 30 : Daimler

MIB 40 : UniCredit

Bomhard von Nikolaus

1956 Allemagne, RFA

DAX 30 : Munich Re

MIB 40 : UniCredit

\section{Bouillot Isabelle 우}

1949 France

BEL 20 : Umicore

CAC 40 : Accor, Saint-

Gobain

\section{Bourdais de}

Charbonnière Eric

1939 France

AEX 25 : ING

CAC 40 : Michelin

Bufe Uwe-Ernst

1944 Allemagne, RFA

AEX 25 : Akzo Nobel

BEL 20 : Solvay, Umicore DAX $30: K+S$

\section{Cardoso Aldo}

1956 France

BEL 20 : Mobistar

CAC 40 : Accor, GDF-

SUEZ

\section{Carron René}

1942 France

CAC 40 : Crédit Agricole, GDF-SUEZ, Lagardère MIB 40 : Fiat

\section{Castaigne Robert}

1946 France

BEL 20 : Nat. Port.

CAC 40 : Sanofi-Aventis, Vinci

\section{Cordero di Montezemolo} Luca

1947 Italie

CAC 40 : PPR

MIB 40 : Fiat

Cremers Frans J. G. M.

1952 Pays-Bas

AEX 25 : Fugro

CAC 40 : Unibail-

Rodamco SA

\section{Cromme Gerhard}

1943 Allemagne, RFA

CAC 40 : Saint-Gobain

DAX 30 : Allianz SE,

Siemens, ThyssenKrupp

\section{Cucchiani Enrico}

Tommaso

1950 Italie

DAX 30 : Allianz SE

MIB 40 : Pirelli, UniCredit 
Répertoire des interlockers transnationaux (suite)

Della Valle Diego

1953 Italie

CAC $40:$ LVMH

MIB 40 : Generali

Demuynck Guido J. M.

1951 Belgique

AEX 25 : TomTom

BEL 20 : Belgacom

Desmarais Jr Paul

1954 Canada

BEL $20: G B L$

CAC 40 : GDF-SUEZ,

Lafarge, Total

d'Este Lorenz

1955 Autriche

BEL $20: U C B$

CAC $40:$ Suez

Environnement

\section{Dunn Douglas}

1944 Royaume-Uni

AEX 25 : TomTom

MIB 40 :

STMicroelectronics

Folz Jean-Martin

1947 France

BEL 20 : Solvay

CAC 40 : Alstom, AXA,

Carrefour, Saint-Gobain,

Société Générale

Frère Albert

1926 Belgique

BEL 20 : GBL

CAC 40 : GDF-SUEZ, LVMH

\section{Frère Gérald}

1951 Belgique

BEL 20 : GBL, Nat. Port.

CAC 40 : Lafarge

Galateri di Genola
Gabriele
1947 Italie
CAC 40 : Accor
MIB 40 : Generali,
Telecom Italia
Goblet d'Alviella Richard
1948 Belgique
BEL 20 : Delhaize,
Danone, GDF-SUEZ

Grube Rüdiger

1951 Allemagne, RFA

CAC 40 : EADS

DAX 30 : Daimler

Harris Mary 우

1966 Royaume-Uni

AEX 25 : TNT

CAC 40 : Unibail-

Rodamco $S A$

Hessels Jan-Michiel

1942 Pays-Bas

AEX 25 : Heineken,

Philips

BEL 20 : Fortis

Hughes Louis R.

1949 Etats-Unis

d'Amérique

AEX 25 : Akzo Nobel

CAC 40 : Alcatel Lucent

Job Peter

1941 Royaume-Uni

AEX 25 : Shell

DAX 30 : Deutsche Bank

Kessler Denis

1952 France

BEL 20 : Dexia

CAC 40 : BNP Paribas
Kley Max Dietrich

1940 Allemagne, RFA

DAX 30 : BASF, Infineon

MIB 40: UniCredit

\section{Kopp Cathy 우}

1949 France

BEL 20 : Dexia

CAC 40 : Schneider

\section{Krebs Rolf}

1940 Allemagne, RFA

CAC 40 : Air Liquide

DAX 30 : E. Merck KGaA

\section{Lagardère Arnaud}

1961 France

CAC 40 : EADS,

Lagardère, LVMH

DAX 30 : Daimler

Lamberti Hemann-Josef

1956 Allemagne, RFA

CAC 40 : EADS

DAX 30 : Deutsche Bank,

Deutsche Börse

\section{Landau Igor}

1944 France

CAC 40 : Sanofi-Aventis

DAX 30 : Adidas, Allianz

SE

\section{Lombard Didier}

1942France

CAC 40 : France Telecom

MIB 40 :

STMicroelectronics

Lombard Marie-

\section{Christine 우}

1958 France

AEX 25 : TNT

DAX 30 : Metro 
Répertoire des interlockers transnationaux

(suite)

Luther Siegfried

1944 Allemagne, RFA

BEL 20 : Nat. Port.

DAX 30 : Infineon

Mangold Klaus

1943 Allemagne, RFA

CAC 40 : Alstom

DAX 30 : Metro

\section{Müller Klaus-Peter}

1944 Allemagne, RFA

DAX 30 : Commerzbank, Linde

MIB 40 : Generali

\section{Mussari Giuseppe}

1962 Italie

CAC 40 : AXA

MIB $40: B M P S$

\section{Polet Robert}

1955 Pays-Bas

AEX 25 : Reed Elsevier

CAC $40: P P R$

\section{Quinet Alain}

1961 France

BEL 20 : Dexia

CAC 40 : Accor

Romanet de Beaune de Augustin

1961 France

BEL 20 : Dexia

CAC 40 : Accor, Veolia

\section{Rudder de Thierry}

1949 Belgique France

BEL 20 : GBL, Nat. Port.

CAC 40 : GDF-SUEZ,

Lafarge, Total

Scaroni Paolo

1946 Italie

CAC 40 : Veolia

MIB 40 : Eni, Generali
Schinzler Hans-Jürgen

1940 Allemagne, RFA

DAX 30 : Metro, Munich

Re

MIB 40 : UniCredit

Segundo de Karen 우

1946 Pays-Bas

AEX 25 : Ahold

DAX 30 : E.ON

Sèze de Amaury

1946 France

BEL 20 : GBL

CAC 40 : Carrefour, Suez

Environnement

Storm Kornelis J.

1942 Pays-Bas

AEX 25 : Aegon, Unilever

Group

BEL 20:AB InBev

\section{Streiff Christian}

1954 France

CAC 40 : PSA

DAX 30 : ThyssenKrupp

\section{Strutz Eric}

1964 Allemagne, RFA

DAX 30 : Commerzbank

MIB 40 : Mediobanca

\section{Thielen Gunter}

1942 Allemagne, RFA

BEL 20 :GBL

CAC 40 : Sanofi-Aventis

\section{Thierry Jean-Philippe}

1948 France

CAC 40 : PPR

DAX 30 : Allianz SE

\section{Uebber Bodo}

1959Allemagne, RFA

CAC 40 : EADS

DAX 30 : Daimler van Lede Cornelis J.A.

1942 Pays-Bas

AEX 25 : Heineken,

Philips

CAC 40 : Air France-KLM, Air Liquide

Van Miert Karel A.L.M.

1942 Belgique

AEX 25 : Philips

BEL 20 : Agfa Gevaert,

Solvay

CAC 40 : Vivendi

DAX 30 : Munich Re,

RWE

van Wijk Leo M.

1946 Pays-Bas

AEX 25 : Aegon,

Randstad Holding

CAC 40 : Air France-KLM

Vaucleroy de Jacques $M$.

1961 Belgique

AEX 25 : ING

BEL 20 : Delhaize

\section{Vuursteen Karel}

1941 Pays-Bas

AEX 25 : Akzo Nobel,

ING,TomTom

DAX 30 : Henkel

Westerburgen Jos W. B.

1942 Pays-Bas

AEX 25 : ASML Holding

CAC 40 : Unibail-

Rodamco SA

Wyand Anthony

1943 Royaume-Uni

Canada

CAC 40 : Société

Générale

MIB 40 : UniCredit 